DOI: https://doi.org/10.34069/RA/2020.6.04

Volumen 3, Número 6/julio-diciembre 2020

\title{
Panorama de la implementación de los sistemas de gestión ambiental en la Amazonia Colombiana
}

\author{
State of the implementation of the environmental managment systems in the \\ Colombian Amazon
}

\section{Visão geral da implementação de sistemas de gestão ambiental na Amazônia colombiana}

Recibido: 12 de septiembre de $2020 \quad$ Aceptado: 1 de diciembre de 2020

\author{
Autores: \\ Magda Julissa Rojas-Bahamón ${ }^{5}$ \\ Diego Felipe Arbeláez-Campillo ${ }^{6}$
}

\section{Resumen}

Este artículo muestra el panorama de aplicación de los sistemas de gestión ambiental en las empresas, fincas, instituciones del Estado, instituciones educativas, la cultura y la economía en el departamento del Caquetá, ubicado en la Región Amazónica colombiana.

La transcendencia del estudio se valora en los aportes que el proceso investigativo hizo en torno al análisis documental de las normas ISO 14001:2014, RAS (Red de agricultura sostenible) y OHSAS 18001, así como la indagación en diferentes empresas privadas, entidades del Estado, instituciones educativas y fincas del departamento del Caquetá sobre la aplicabilidad de este tipo de normas en la construcción de los sistemas de gestión ambiental.

El estudio permitió vislumbrar que en el departamento del Caquetá el panorama de aplicación de estándares internacionales sobre SGA es escaso, debido mayormente al desconocimiento de la norma por parte de las organizaciones locales. Así mismo, que es necesaria la implementación de estos sistemas para lograr procesos organizados y de calidad.

Palabras claves: Sistemas de gestión ambiental, ISO 14001:2014, RAS, Caquetá.

\begin{abstract}
This paper shows the panorama of application of environmental management systems in companies, farms, State institutions, educational institutions, culture and the economy in the department of Caquetá, located in the Colombian Amazonia.

The significance of the study is valued in the contributions that the investigative process made regarding the documentary analysis of the ISO 14001: 2014, RAS (Sustainable Agriculture Network) and OHSAS 18001 standards, as well as the investigation in different private companies, State entities, educational institutions and farms of the department of Caquetá on the applicability of this type of norms in the construction of environmental management systems.

The study allowed us to glimpse that in the department of Caquetá the panorama of application of international standards on EMS is scarce, mainly due to the ignorance of the norm by local

\footnotetext{
${ }^{5}$ PhD. Educación y Cultura Ambiental. Docente titular Secretaría Educación Florencia. Par evaluadora Colciencias Colombia. ORCID ID: https://orcid.org/0000-0003-4882-1476

6 Grupo de investigación Lenguajes, representaciones y Educación, Universidad de la Amazonia (Colciencias- Colombia). ORCID ID: https://orcid.org/0000-0002-9041-9563
} 
organizations. Likewise, the implementation of these systems is necessary to achieve organized and quality processes.

Key Words: Environmental management systems, ISO 14001: 2014, RAS, Amazonia colombiana, Caquetá.

\section{Resumo}

Este artigo mostra o panorama da aplicação dos sistemas de gestão ambiental em empresas, fazendas, instituições do Estado, instituições de ensino, cultura e economia no departamento de Caquetá, localizado na Amazônia colombiana.

A significância do estudo é avaliada nas contribuições que o processo investigativo fez no que diz respeito à análise documental das normas ISO 14001: 2014, RAS (Rede de Agricultura Sustentável) e OHSAS 18001, bem como na investigação em diferentes empresas privadas, entidades do Estado, instituições de ensino e fazendas do departamento de Caquetá sobre a aplicabilidade desse tipo de normas na construção de sistemas de gestão ambiental.

O estudo permitiu vislumbrar que no departamento de Caquetá o panorama de aplicação das normas internacionais sobre SGA é escasso, principalmente devido ao desconhecimento da norma por parte das organizações locais. Da mesma forma, a implantação desses sistemas é necessária para a obtenção de processos organizados e de qualidade.

Palavras-chave: Sistemas de gestão ambiental, ISO 14001: 2014, RAS, Caquetá.

\section{Introducción}

Un sistema de gestión ambiental (SGA) es una herramienta cuya implantación persigue mejorar el comportamiento ambiental de una empresa. Un SGA se constituye a base de acciones medioambientales y elementos de gestión.

De esta manera, este documento tiene como propóstio presentar los impactos que los estándares internacionales sobre sistemas de gestión ambiental generan sobre las empresas, las fincas, las instituciones del Estado, la educación, la cultura y la economía en el departamento del Caquetá, Colombia.

Para lo anterior, el estudio abordó el análisis documental de las normas ISO 14001:2014, RAS (Red de agricultura sostenible) y OHSAS 18001, así como la indagación de la aplicabilidad de los estándares en diferentes empresas privadas, entidades del Estado, instituciones educativas y fincas del departamento del Caquetá, a fin de determinar los impactos potenciales.

Para este estudio, de tipo descriptivo y de carácter mixto, se utilizó una metodología que involucró técnica cuantitativas y cualitativas. Desde el ámbito cualitativo se aplicó la técnica de análisis documental y entrevistas; desde lo cualitativo, el estudio involucró la aplicación de una encuesta para determinar la existencia o no de un sistema de gestión ambiental o una política ambiental en la organización.

En los resultados del proceso de indagación se obtuvo información sobre la aplicabilidad de las normas internacionales sobre sistemas de gestión ambiental en fincas, en diferentes empresas públicas, privadas y educativas del departamento de Caquetá, así como la determinación de los impactos potenciales de dichas normas sobre los la economía y la cultura.

El documento aborda entonces referentes teóricos respecto a los sistemas de gestión ambiental (SGA) desde los estándares internacionales ISO 14001, RAS y OHSAS 1800; antecendentes investigativos en Colombia respecto a impacto de los estándares internacionales; 


\section{AMAZONANAS}

la descripción de la metodología utilizada para el estudio; los resultados en términos del discurso de convergencia de los estándares internacionales sobre SGA y la información de los SGA con certificación internacional existentes en el departamento del Caquetá.

Luego, se presenta el apartado de discusión, en el que se establece el impacto potencial de la estandarización en SGA en las empresas y fincas caqueteñas y economía del país, en la educación y en la cultura. Finalmente se abordan las conclusiones del estudio y la bibliografía.

\section{Los sistemas de gestión ambiental y estándares internacionales}

Con un entorno económico caracterizado por la globalización e integración de los mercados, los procesos de normalización se han convertido en un mecanismo de coordinación y un instrumento de regulación eficaces para la gestión empresarial, de esta manera los sistemas de gestión están basados en la formalización de las actividades y procesos, lo cual conlleva a su estandarización. Lannelongue, Gonzalez, y Gonzalez (2011)

De acuerdo con lo mencionado, en Colombia, mediante la Ley 99 de 1993, se creó el Ministerio de Ambiente, para el manejo ambiental, y se establecieron las bases del Sistema Nacional Ambiental (SINA), como el conjunto de principios, orientaciones, normas, actividades, recursos, programas e instituciones o entidades responsables de la política ambiental que permiten la puesta en marcha de los principios generales ambientales.

Así mismo, entre las funciones del ministerio de ambiente, está la formulación de la política nacional en relación con el medio ambiente y los recursos naturales renovables, así como establecer las reglas y criterios de ordenamiento ambiental, para asegurar el aprovechamiento sostenible de los recursos naturales renovables y del medio ambiente, conjuntamente con el Ministerio de Salud, el Ministerio de Desarrollo Económico, la política nacional con el Ministerio de Agricultura, con el Ministerio de Comercio Exterior, con el Ministerio de Educación Nacional. En consecuencia, según Varela (2009) la protección y recuperación del medio ambiente es una tarea conjunta del Estado, la comunidad, las ONG y el sector privado, por lo tanto, un Sistema de Gestión Ambiental (SGA) es un sistema de procesos y prácticas que permiten a una organización reducir sus impactos sobre el medio ambiente y aumentar su eficacia de funcionamiento.

De acuerdo con lo mencionado, se considera que un aspecto fundamental de la gestión ambiental, es generar una actitud preventiva para identificar afectaciones ambientales negativas de políticas, planes y proyectos con el propósito de eliminarlas o reducirlas a niveles aceptables.

\section{Norma ISO 14000}

De acuerdo con el Foro Nacional Ambiental realizado en Bogotá en el 2011, la inquietud por la protección de la salud de los humanos y la responsabilidad social, han sido preocupaciones prioritarias para las naciones industrializadas en el mundo, en los últimos treinta años. En este sentido, en la vía de lo plantado por Miranda, Toro y Agudelo, (2010), la organización internacional de Normalización ISO, es el organismo encargado de promover el desarrollo de normas internacionales de fabricación, comercio y comunicación para todas las ramas industriales a excepción de la eléctrica y la electrónica y fue en la Cumbre de la Tierra de 1992, donde adquirió el compromiso de crear normas internacionales para la gestión ambiental empresarial.

Así, la serie de normas ISO 14000 es el producto del compromiso de establecer un indicador global que compromete a las organizaciones con el desarrollo sostenible. Es de aclarar que esta norma no tiene carácter obligante, de tal manera que los países que forman parte de la ISO, la acogen y son las empresas y las organizaciones quienes la aplican voluntariamente, puesto 
que la norma brinda herramientas metodológicas para la implementación de sistemas de gestión ambiental en procesos de producción.

Por lo anterior, Torres (2009) afirma es necesaria la aplicación de normas ISO y producción más limpia, para obtención de la licencia ambiental, aunque según Méndez (2009) una organización puede implantar un sistema de gestión ambiental y no requerir necesariamente que una organización externa lo certifique; sin embargo, por lo general las organizaciones suelen acudir a un organismo certificador que confirme la adecuación de su sistema con la norma ISO 14000.

Tabla 1.

Descripción de normas ISO 14000

\begin{tabular}{|c|c|}
\hline $\begin{array}{l}\text { NORMA } \\
\text { ISO } 14000\end{array}$ & APORTE \\
\hline $\begin{array}{c}\text { Ley } 14004 \\
\text { de } 2004\end{array}$ & $\begin{array}{l}\text { Proporciona asistencia a las organizaciones que deseen implementar o mejorar un } \\
\text { sistema de gestión ambiental y con ello mejorar su desempeño ambiental y aunque el } \\
\text { sistema no está previsto para gestionar temas de salud ocupacional y seguridad, estos } \\
\text { pueden estar incluidos cuando una organización busca implementar un sistema } \\
\text { integrado de gestión ambiental, salud y seguridad ocupacional. }\end{array}$ \\
\hline 14001.1996. & $\begin{array}{l}\text { Especifica los requisitos de un sistema de administración ambiental, no está previstas } \\
\text { para crear barreras arancelarias al comercio, ni para aumentar o cambiar las } \\
\text { obligaciones legales de una organización, es aplicable a organizaciones de todo tipo y } \\
\text { tamaño, y puede acomodarse a diversas condiciones geográficas, culturales y sociales. }\end{array}$ \\
\hline 14010 & $\begin{array}{l}\text { Es una guía para las organizaciones, los auditores y sus clientes sobre los principios } \\
\text { comunes en pro de la ejecución de las auditorías ambientales. }\end{array}$ \\
\hline 14011 & $\begin{array}{l}\text { Presenta los procedimientos requeridos en auditorías de sistemas de administración } \\
\text { ambiental. }\end{array}$ \\
\hline 14012 & $\begin{array}{l}\text { Guía sobre los criterios de calificación para los auditores ambientales con el fin de } \\
\text { apoyar la aplicación de los sistemas de administración ambiental y las auditorías } \\
\text { ambientales. }\end{array}$ \\
\hline 14020 & $\begin{array}{l}\text { Establece principios para el desarrollo y uso de los sellos y declaraciones ambientales. } \\
\text { La información sobre los aspectos ambientales de productos y servicios, pertinentes al } \\
\text { sello o declaración ambiental, debe estar a disposición de los compradores reales y } \\
\text { potenciales de la parte que hace la declaración o sello ambiental. }\end{array}$ \\
\hline 14021 & $\begin{array}{l}\text { Esta norma especifica requisitos para auto declaraciones ambientales en cuanto a } \\
\text { productos, incluyendo afirmaciones, símbolos y gráficos, también describe una } \\
\text { metodología general para la evaluación y la verificación de las auto declaraciones } \\
\text { ambientales, así como métodos específicos de evaluación y verificación para las } \\
\text { declaraciones seleccionadas en esta norma. }\end{array}$ \\
\hline 14031 & $\begin{array}{l}\text { Brinda orientación sobre el diseño y empleo de la evaluación del desempeño ambiental } \\
\text { dentro de una organización. No establece niveles de desempeño ambiental. }\end{array}$ \\
\hline 14024 & $\begin{array}{l}\text { Esta norma establece los principios y procedimientos para desarrollar programas de eco- } \\
\text { rotulado Tipo 1, incluyendo la selección de las categorías de producto, criterios } \\
\text { ambientales de producto y características de función del producto; para evaluar y } \\
\text { demostrar conformidad }\end{array}$ \\
\hline 14040 & $\begin{array}{l}\text { Esta norma establece la estructura general, los principios y los requisitos generales para } \\
\text { conducir y reportar estudios de evaluación del ciclo de vida. }\end{array}$ \\
\hline 14041 & $\begin{array}{l}\text { Describe los requisitos y los procedimientos, necesarios para la compilación y la } \\
\text { preparación de la definición del propósito y del alcance para una evaluación de ciclo de } \\
\text { vida, y la realización, interpretación y el informe de un análisis de inventario. }\end{array}$ \\
\hline 14043 & $\begin{array}{l}\text { Describe la fase final del procedimiento de evaluación del ciclo de vida (ECV), en la } \\
\text { cual los resultados de un análisis de inventario de ciclo de vida (ICV), y, si se ha } \\
\text { realizado, de una evaluación del impacto del ciclo de vida (EICV), }\end{array}$ \\
\hline 14045 & $\begin{array}{l}\text { Se presenta el vocabulario relacionado con administración ambiental según las normas } \\
\text { ISO } 14.000\end{array}$ \\
\hline
\end{tabular}




\section{AMAZONANAS}

En Colombia, de acuerdo con Pérez y Bejarano (2007) las certificadoras en ISO acreditadas por la Superintendencia de Industria y Comercio son: EI instituto Colombiano de Normas técnicas-Icontec, Bureau Veritas QualiT y la Societé Generale de Surveillance S.A.SGS. Así mismo, el autor afirma que hay un mayor interés de las empresas grandes por la certificación, seguramente porque cuentan con más recursos, puesto que en la mayoría de los casos se requiere invertir en equipos que permitan el mejoramiento del manejo de residuos sólidos, aguas residuales, manejo de contaminación visual, sonora y atmosférica.

Red de agricultura sostenible RAS.

En un mundo de constante cambio, las tendencias actuales han demostrado que el uso racional de los recursos naturales, un trato justo a los trabajadores y la conservación de la vida silvestre, han dejado de ser valores agregados en la cadena de custodia para convertirse en factores determinantes a la hora de participar activamente en el mercado global. Guzmán y Medina (2012)

En este sentido, la Red de Agricultura Sostenible (RAS) es una coalición de organizaciones independientes en el ámbito internacional, sin fines de lucro que promueve la sostenibilidad ambiental y social de las actividades agrícolas por medio del desarrollo de normas. Es un Organismo de Certificación que legaliza fincas que cumplen con las normas de la RAS; de acuerdo con lo mencionado, la certificación otorgada se denomina Rainforest Alliance, y se sustenta en la protección ambiental, equidad social y viabilidad económica, impulsando mejores prácticas para la cadena de valor agropecuaria para mejorar la calidad de los productos mediante la sostenibilidad.

Según Benoit (2011), el crecimiento de la certificación Rainforest Alliance en Colombia, se genera porque:

"las tendencias del mercado internacional y al interés de los consumidores por adquirir productos obtenidos a partir de una producción Sostenible está es su punto más alto. Para los productores la implementación de la norma ha mejorado sus condiciones de vida, ha animado el desarrollo de las comunidades rurales, ha mitigado el impacto ambiental de los sistemas de producción agrícolas y el bienestar de los trabajadores en nuestro país". (p. 53).

Según la Red de Agricultura sostenible (2013), durante el transcurso de los últimos años, la RAS ha realizado talleres para la interpretación local de la norma para Agricultura Sostenible, cumbres de capacitación para las regiones de África, Asia y América Latina, con los técnicos líderes de la RAS para procesos de auditoría y de asesoría técnica en aras de fomentar el uso racional de los recursos naturales, un trato justo a los trabajadores, la conservación de la vida silvestre y buenas relaciones entre las fincas y sus vecinos, para producir agropecuariamente, conservando la biodiversidad y desarrollo humano sostenible. Así mismo tiene criterios o límites no-negociables, aspecto que demuestra que es una norma muy exigente, pero se puede lograr su cumplimiento en la mayoría de las fincas.

Los criterios de la norma miden el cumplimiento de los diez principios de la agricultura sostenible, los cuales son:

1. Sistemas de gestión social y ambiental

2. Conservación de ecosistemas

3. Protección de la vida silvestre

4. Conservación de recursos hídricos

5. Trato justo y buenas condiciones para trabajadores

6. Salud y seguridad ocupacional

7. Relaciones con la comunidad

8. Manejo integrado de cultivo 


\section{AMAZONAS}

9. Manejo y conservación de suelo

10. Manejo integrado de desechos.

De acuerdo con lo mencionado, el objetivo de la norma es alentar a las fincas a analizar y por consiguiente mitigar los riesgos ambientales y sociales causados por actividades de la agricultura por medio de un proceso que motiva el mejoramiento continuo, que busca integrar la producción agropecuaria sostenible, aumentar la conciencia de agricultores, comercializadores, consumidores e industrias, y facilitar foros de discusión entre grupos ambientales. En consecuencia, la norma para Agricultura Sostenible incorpora prácticas que reducen las emisiones de gases de efecto invernadero (GEI), promoviendo reservas de carbono en las fincas y ayudando a los productores a aumentar la resiliencia de sus fincas frente al cambio climático.

\section{Norma internacional OHSAS 18001}

Las organizaciones de todo tipo están cada vez más preocupadas por lograr y demostrar un desempeño sólido en cuanto a seguridad y salud ocupacional ( $\mathrm{S}$ y $\mathrm{SO}$ ) mediante el control de sus riesgos, en coherencia con su política y objetivos de S y SO, todo esto dentro del contexto de una legislación cada vez más estricta, el desarrollo de políticas económicas y otras medidas que fomenten buenas prácticas de $\mathrm{S}$ y SO, y la creciente preocupación expresada por las partes interesadas acerca de estos aspectos.

En este sentido, como requisito, la organización debe establecer, implementar y mantener procedimientos para la identificación y acceso a requisitos legales, así mismo debe establecer, implementar y mantener documentados los objetivos de S y SO en las funciones y niveles pertinentes dentro de la organización.

En consecuencia, se habla de competencia, formación y toma de conciencia, dado que la organización debe asegurar que cualquier persona que esté bajo su control ejecutando tareas que pueden tener impacto sobre la S y SO, sea competente con base en su educación, formación o experiencia, y debe conservar los registros apropiados. La organización debe asegurarse de que las auditorías internas del sistema de gestión de S y SO se lleven a cabo. De igual manera, la alta dirección, debe revisar, a intervalos definidos, el sistema de gestión de S y SO para asegurar su conveniencia, adecuación y eficacia permanente. Las revisiones deben incluir las oportunidades de evaluación para la mejora, y la necesidad de cambios al sistema de gestión de S y SO, incluida la política y los objetivos de S y SO.

\section{Antecedentes investigativos en Colombia.}

A continuación, se presenta una tabla con la información de los antecedentes investigativos en Colombia en relación con los impactos de los estándares de gestión ambiental clasificadas desde diversas categorías: empresas, fincas, sector educativo e instituciones públicas. 
Tabla 2.

Antecedentes investigativos en Colombia sobre impacto estándares de Gestión Ambiental.

\begin{tabular}{|c|c|c|c|}
\hline \multicolumn{4}{|c|}{ EMPRESAS } \\
\hline INVESTIGACIONES & IMPACTO ESTÁNDARES G.A. & $\begin{array}{c}\text { AUTOR } \\
\text { (ES) }\end{array}$ & AÑ̃ \\
\hline $\begin{array}{l}\text { Evaluación del } \\
\text { impacto de la } \\
\text { cooperación } \\
\text { internacional para el } \\
\text { desarrollo en la } \\
\text { gestión ambiental }\end{array}$ & $\begin{array}{l}\text { Las empresas tienen responsabilidad en la gestión } \\
\text { ambiental, en mejoramiento de procesos, } \\
\text { productos para disminuir impactos ambientales. } \\
\text { La Política Ambiental en Colombia tiene tres } \\
\text { fuentes principales de financiación: i) las rentas } \\
\text { propias, ii) el presupuesto general de la nación } \\
\text { (PGN), y iii) la Cooperación Internacional. }\end{array}$ & $\begin{array}{l}\text { Miranda, P., } \\
\text { Toro, J. y } \\
\text { Agudelo, J. }\end{array}$ & 2010 \\
\hline $\begin{array}{l}\text { Licencias } \\
\text { ambientales y su } \\
\text { proceso de } \\
\text { reglamentación en } \\
\text { Colombia. }\end{array}$ & $\begin{array}{l}\text { La función de las normas ISO, es la de buscar la } \\
\text { estandarización de normas de productos y } \\
\text { seguridad para las empresas u organizaciones a } \\
\text { nivel internacional. } \\
\text { Las políticas de desarrollo económico del país, } \\
\text { que sirven de argumento principal para la } \\
\text { aprobación de muchos proyectos, unidas al } \\
\text { proceso de gestión de calidad, han ocasionado que } \\
\text { la autoridad ambiental dirija sus tareas en la } \\
\text { expedición rápida de licencias ambientales con un } \\
\text { deficiente proceso de evaluación y dejando a un } \\
\text { lado su misión como protectora del ambiente. }\end{array}$ & $\begin{array}{l}\text { FNA. Foro } \\
\text { Nacional } \\
\text { Ambiental. }\end{array}$ & 2011 \\
\hline $\begin{array}{l}\text { Implementación del } \\
\text { sistema de gestión } \\
\text { ambiental (SGA) } \\
\text { bajo la norma NTC- } \\
\text { ISO } 14001 \text { en el } \\
\text { proceso industrial } \\
\text { del arroz en la } \\
\text { arrocera la } \\
\text { esmeralda } \text { S. A. }\end{array}$ & $\begin{array}{l}\text { La implementación del Sistema de Gestión } \\
\text { Ambiental, sirvió para desarrollar fortalezas en } \\
\text { requisitos legales que estaban en incumplimiento. } \\
\text { Se debe mantener y sostener el Sistema de Gestión } \\
\text { Ambiental NTC-ISO 1400-1, llevando a cabo } \\
\text { todos los procedimientos y formatos establecidos; } \\
\text { ya que estos son fundamentales para que el } \\
\text { sistema pueda operar sin errores. }\end{array}$ & Arcila Diego & 2011 \\
\hline \multicolumn{4}{|c|}{ FINCAS } \\
\hline INVESTIGACIONES & IMPACTO ESTÁNDARES G.A. & $\begin{array}{c}\text { AUTOR } \\
(\mathbf{E S})\end{array}$ & AÑO \\
\hline $\begin{array}{l}\text { Formulación para la } \\
\text { implementación de } \\
\text { la norma para la } \\
\text { agricultura } \\
\text { sostenible de la RAS } \\
\text { - sello Rainforest } \\
\text { Alliance, para la } \\
\text { finca cafetera "la } \\
\text { reina" perteneciente } \\
\text { a la } \\
\text { comercializadora } \\
\text { internacional } \\
\text { compañía cafetera } \\
\text { agrícola de } \\
\text { Santander S.A (CI } \\
\text { CIA CCA S.A) en el } \\
\text { municipio la } \\
\text { Virginia (Risaralda). }\end{array}$ & $\begin{array}{l}\text { El desarrollo e implementación de todos los } \\
\text { programas, actividades y planes de mejora para la } \\
\text { finca, han generado un importante avance, } \\
\text { generando un impacto altamente positivo no sólo } \\
\text { a nivel operacional sino también en todos sus } \\
\text { colaboradores, incluidos los responsables del } \\
\text { presente proyecto, perfilando así a La Reina como } \\
\text { un ejemplo no sólo para las otras fincas de la } \\
\text { compañía sino además de la región y del país. }\end{array}$ & $\begin{array}{l}\text { Guzmán, D. } \\
\text { y Medina, J. }\end{array}$ & 2012 \\
\hline
\end{tabular}




\begin{tabular}{|c|c|c|c|}
\hline \multicolumn{4}{|c|}{ EDUCACIÓN } \\
\hline $\begin{array}{c}\text { INVESTIGACIONES } \\
\text {. }\end{array}$ & IMPACTO ESTÁNDARES G.A. & $\begin{array}{c}\text { AUTOR } \\
\text { (ES) }\end{array}$ & AÑ̃ \\
\hline $\begin{array}{l}\text { La política ambiental } \\
\text { en los planes de } \\
\text { desarrollo en } \\
\text { Colombia 1990-2006. }\end{array}$ & $\begin{array}{l}\text { Colombia ha sido uno de los primeros países de } \\
\text { América Latina en contar con una normatividad } \\
\text { sobre el manejo y protección de los recursos } \\
\text { naturales y el medio ambiente. } \\
\text { La gestión pública es fundamental para regular y } \\
\text { controlar el uso de los recursos naturales y las } \\
\text { funciones ambientales y así garantizar la } \\
\text { sostenibilidad ambiental y la calidad de vida. Los } \\
\text { Estados materializan estos propósitos en } \\
\text { instrumentos de política, que pueden ser de } \\
\text { regulación directa, administrativos y de } \\
\text { planificación, económicos y de educación, } \\
\text { información, investigación y asistencia técnica. }\end{array}$ & $\begin{array}{l}\text { Tobasura } \\
\text { Isaías. }\end{array}$ & 2006 \\
\hline $\begin{array}{l}\text { Proyecto educativo } \\
\text { del programa. }\end{array}$ & $\begin{array}{l}\text { Obtención junto con la titulación como } \\
\text { Especialista en Gestión Ambiental, una } \\
\text { certificación como auditor interno en Sistemas } \\
\text { de Gestión Integrados (ISO 9001-ISO 14001- } \\
\text { OHSAS 18001) }\end{array}$ & $\begin{array}{l}\text { Universidad } \\
\text { de América. }\end{array}$ & 2013 \\
\hline \multicolumn{4}{|c|}{ INSTITUCIONES PUBLICAS } \\
\hline INVESTIGACIONES & IMPACTO ESTÁNDARES G.A. & $\begin{array}{c}\text { AUTOR } \\
\text { (ES) }\end{array}$ & AÑO \\
\hline $\begin{array}{l}\text { Documento base para } \\
\text { la formulación, } \\
\text { concertación, } \\
\text { implementación, } \\
\text { evaluación, control y } \\
\text { seguimiento del plan } \\
\text { institucional de } \\
\text { gestión ambiental - } \\
\text { PIGA. }\end{array}$ & $\begin{array}{l}\text { El Plan Institucional de Gestión Ambiental - } \\
\text { PIGA - es el instrumento de planeación ambiental } \\
\text { a corto plazo, que parte del análisis de la situación } \\
\text { ambiental institucional, con el propósito de } \\
\text { brindar información y argumentos necesarios para } \\
\text { el planteamiento de acciones de gestión ambiental } \\
\text { que garanticen el cumplimiento de los objetivos } \\
\text { de ecoeficiencia establecidos en el Decreto } 456 \text { de } \\
2008 \text {, entre otras acciones ambientales que } \\
\text { contemplen las entidades y aporten a la totalidad } \\
\text { de los objetivos ambientales establecidos en el } \\
\text { PGA. } \\
\text { Se requiere la estandarización del instrumento de } \\
\text { planeación y gestión ambiental por parte de las } \\
\text { entidades tanto públicas distritales como } \\
\text { voluntarias y, del trabajo conjunto de todos los } \\
\text { actores involucrados en la administración Distrital } \\
\text { de Bogotá. } \\
\text { De esta manera se pretende avanzar hacia la } \\
\text { adopción e implementación de sistemas } \\
\text { integrados de gestión, que, en materia ambiental, } \\
\text { se basan en la norma técnica NTC-ISO 14001; y } \\
\text { que se debe realizar de manera gradual conforme } \\
\text { a la evolución del instrumento en las entidades. }\end{array}$ & $\begin{array}{l}\text { Secretaría } \\
\text { distrital de } \\
\text { ambiente. } \\
\text { Subdirección } \\
\text { de políticas y } \\
\text { planes } \\
\text { ambientales }\end{array}$ & 2014 \\
\hline
\end{tabular}

\section{Método}

Este estudio es de tipo descriptivo y de carácter mixto, puesto que involucra técnica cuantitativas y cualitativas. La metodología cualitativa involucró la aplicación de la técnica de 


\section{AMAZÉNAS}

análisis documental ${ }^{7}$ y entrevistas; desde el ámbito cuantitativo, el estudio involucró la aplicación de una encuesta para determinar la existencia o no de un sistema de gestión ambiental o una política ambiental en la organización.

Para el análisis documental se tuvo como objeto de estudio los estándares internacionales ISO 140001, RAS (Red de agricultura sostenible) y OHSA 18001 (Occupational Health and Safety Assessment Series).

En el proceso de análisis documental se utilizó el software NVivo ${ }^{8}$ el cual permitió organizar y acceder fácilmente al conocimiento conceptual de los estándares, así como identificar tendencias e ideas comunes en los documentos en torno a la constitución de un sistema de gestión ambiental. El análisis se hizo con base en las gráficas del mapa de frecuencia de palabras y la marca de nube de palabras en los documentos.

Para la indagación sobre la existencia de sistemas de gestión ambiental o en su defecto, políticas ambientales en diferentes entidades del departamento del Caquetá, así como la normatividad aplicada para la constituciones de dichos sistemas de gestión, se realizaron entrevistas a los directivos de las empresas, a los encargados del área administrativa y los jefes de control interno.

Para las entrevistas se tomó el texto como objeto de análisis, de manera que se asumió la narrativa como eje principal del discurso en las entrevistas realizadas.

La selección de la unidad de análisis estuvo determinada por organizaciones del sector educativo (instituciones de educación básica y media y educación superior), empresas privadas, empresas públicas, organizaciones de salud, asociaciones y fincas en el municipio Florencia y algunos municipios del Caquetá como San Vicente del Caguán, El Docello, Paujil, Solano, Puerto Rico, Morelia y Belén de los Andaquíes.

\section{Resultados}

\section{El discurso de los estándares internacionales sobre sistemas de gestión ambiental (SGA)}

De manera inicial, la comparación de las normas vistas desde la tabla de contenido, permite identificar que su estructura obedece al mismo esquema, al mismo isomorfismo. En todas las normas, ISO 14001, RAS y OHSAS, el discurso empieza con la determinación de las política, luego se abordan los requisitos, de los cuales se observa que algunos convergen en su sentido de ambientales y de calidad.

El análisis documental de los estándares internacionales sobre sistemas de gestión ambiental (SGA) bajo los parámetros de ISO 140001, RAS y OHSA 18001 abordados en este estudio, permitió vislumbrar puntos convergentes en los documentos. De esta manera, el análisis bajo el software cualitativo permitió establecer que la tendencia de los tres estándares gira en torno a asumir el ambiente y la organización como ejes centrales sobre la cual se teje el discurso de las normas.

\footnotetext{
${ }^{7}$ El análisis documental ha sido considerado como el conjunto de operaciones destinadas a representar el contenido y la forma de un documento para facilitar su consulta o recuperación, o incluso para generar un producto que le sirva de sustituto. (Clauso, 1993)

${ }^{8} \mathrm{NVivo}$ es un software que facilita el manejo de la información para el análisis cualitativo y permite, entre otras tareas, la división de los textos en unidades de significado. (Palacios, Gutiérrez, Sánchez, s.f.)
} 
El esquema de los resultados del análisis documental, visto desde el mapa de nubes de palabras de acuerdo a lo extraído en el software se puede resumir como sigue:

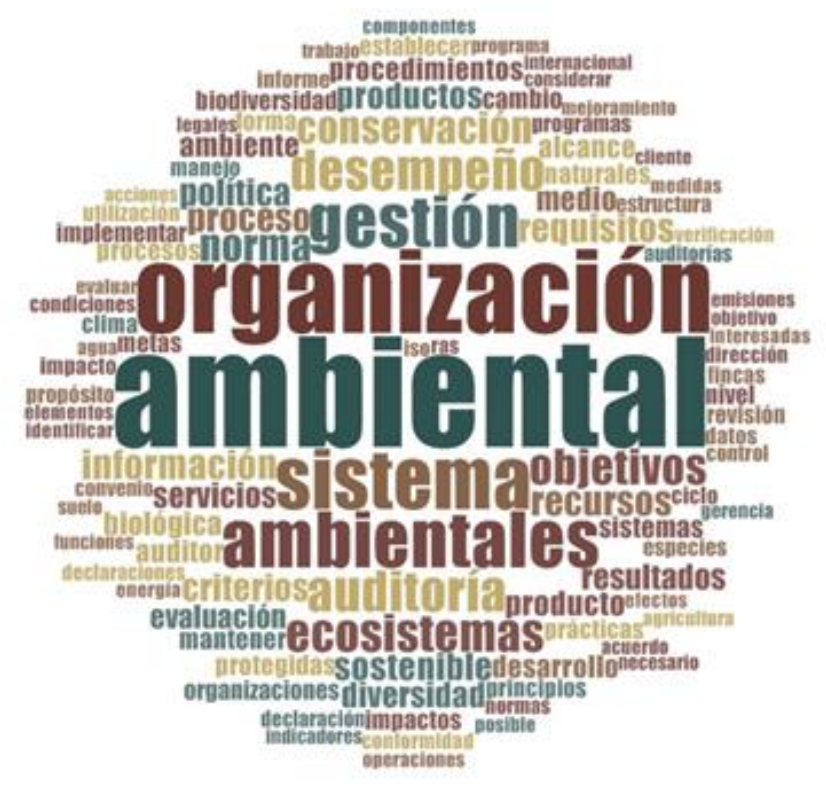

Figura 1. Marca de nube de palabras en documentos de estándares internacionales en sistemas de gestión ambiental

De esta manera, alrededor del discurso de la sinergia entre el ambiente y la organización, se destaca la importancia de la conservación de los recursos naturales, en la cual el agua juega un papel primordial, así como la conservación de los ecosistemas y la sostenibilidad del medio.

El lenguaje común de las normas establece que la conservación ambiental, depende directamente de una estructura organizativa muy sólida, en la cual la auditoría, la verificación constante de las prácticas, la gestión y las políticas de conservación, son el puente que permite lograr la sostenibilidad de los recursos. Así, el constante flujo de información organizacional enmarcados en el logro de los objetivos en torno al tema ambiental, es el foco vital de la norma.

El discurso más lejano de los estándares giran en torno a aspectos de productividad organizacional en la que se consideran criterios como el cliente, las funciones, los datos, los resultados y el alcance e impacto del sistema sobre el ámbito productivo, es decir, la productividad y la competitivdad de la empresa no es el fin de las normas de estándarización en este caso.

\section{Los SGA con certificación internacional en el departamento del Caquetá}

Los resultados de la aplicación de los instrumentos se organizaron por categorías de empresas o instituciones entrevistadas. De esta manera, se establecieron seis grupos: entidades bancarias, entidades de la salud, entidades de educación (que abordó instituciones educativas de educación básica y media, y superior), organizaciones (que involucró asociaciones, ONG e institutos), empresas del sector comercial (de transporte, servicios y productos) y fincas.

La indagación en esta categoría permitió establecer que si bien algunas normas como la ISO 140001es ampliamente conocida y en muchos casos es citada en los documentos institucionales como referente base de la política ambiental, se evidencian pocas certificaciones de la norma en el departamento del Caquetá. 


\section{AMAZÉNAS}

Los resultados de la aplicación de los instrumentos, permitió determinar que el 100\% de las entidades de salud, instituciones educativas y fincas encuestadas en el departamento del Caquetá, no cuentan con SGA bajo la certificación ISO 14001.

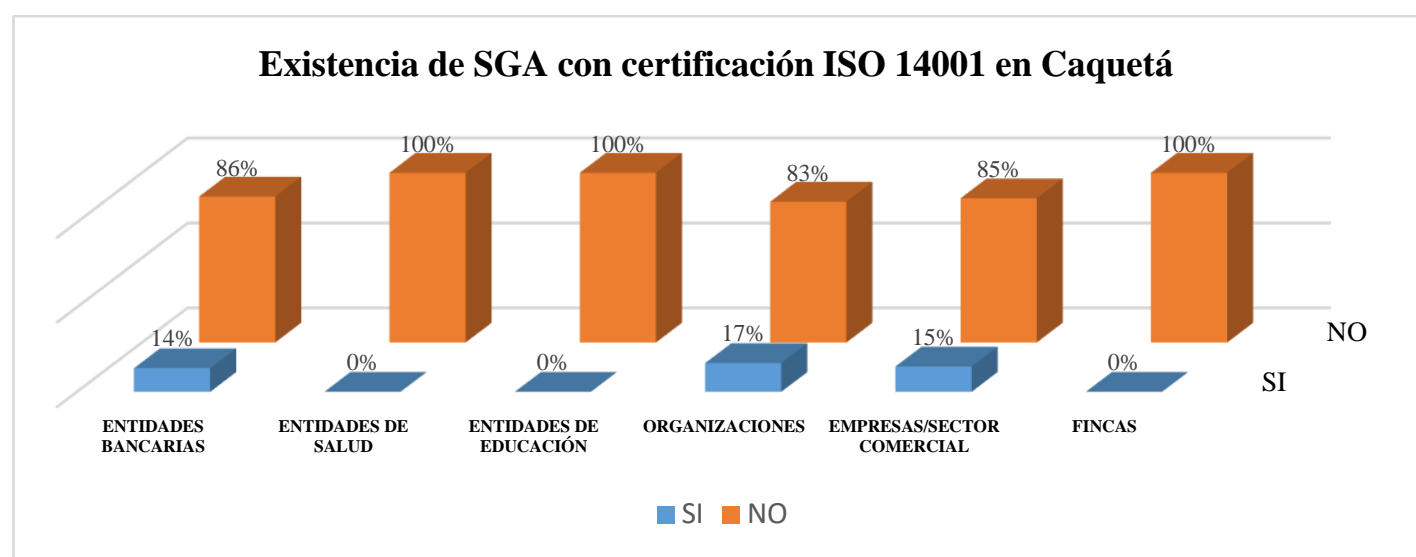

Figura 2. Gráfica de existencia de SGA con certificación ISO 14001 en el departamento del Caquetá.

Igualmente, se encontró en entidades bancarias, empresas del sector comercial y algunas organizaciones (dentro de las que se catalogan institutos, asociaciones) poseen SGA bajo certificación internacional. El cumplimiento de este estándar se presenta debido a que son empresas u organizaciones que hacen parte de multinacionales o empresas muy reconocidas a nivel nacional o internacional, cuyas sedes principales están ubicadas en ciudades capitales, como el caso de Banco BBVA, Avianca o el Instituto Colombiano de Bienestar Familiar (ICBF).

En algunos casos, la información permitió vislumbrar que, si bien algunas sedes de empresas u organizaciones poseen SGA bajo estándar ISO 14001, en las sucursales ubicadas en Florencia, solo existe una política de gestión ambiental que es extendida desde la sede principal.

Las encuestas realizadas en las diferentes organizaciones permitieron vislumbrar que en general, en el departamento del Caquetá, no existe la aplicación de la norma ISO 14001 como base para la implementación de sistemas de gestión ambiental. Sin embargo, la mayoría de las empresas y organizaciones de los diferentes sectores sí cuentan con política ambiental.

Respecto al análisis de la existencia de política ambiental en las diferentes empresas y organizaciones abordadas, se estableció que la totalidad de las entidades bancarias y las entidades de salud poseen política de gestión ambiental enmarcadas dentro de la estrategia de responsabilidad social.

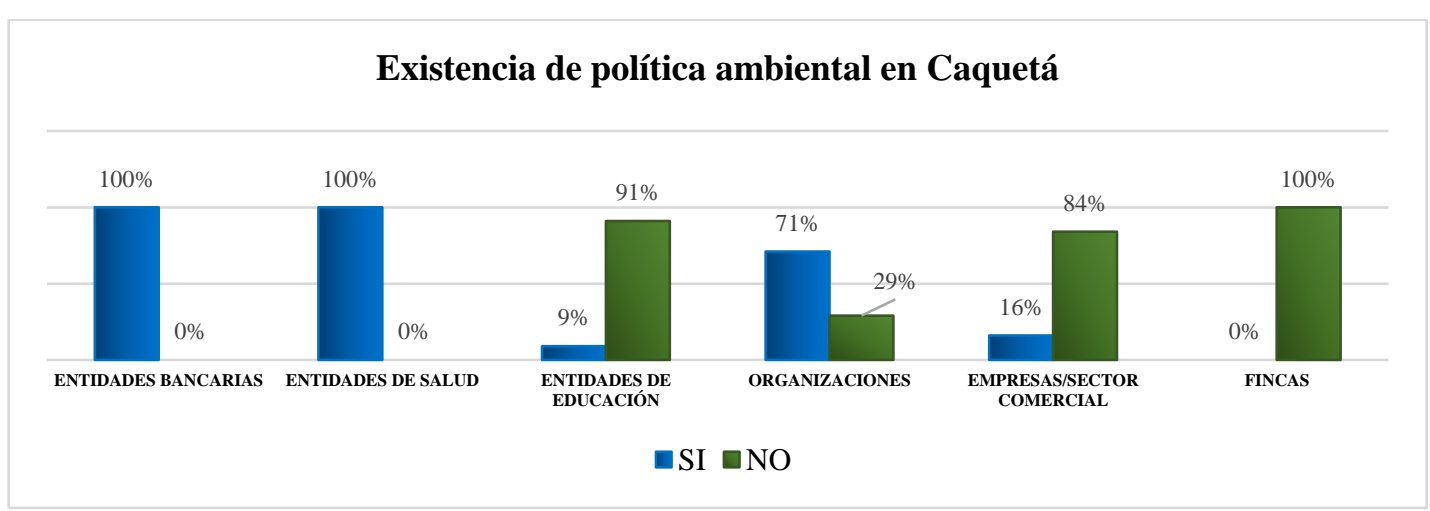

Figura 3. Gráfica de existencia de política ambiental en el departamento del Caquetá. 
Para el caso de las entidades del sector educativo, se encontró en las instituciones públicas de educación básica y media la inexistencia de una política de gestión ambiental, emanada como un acto administrativo legalmente constituido; sin embargo, la totalidad de los entrevistados manifestaron la existencia del proyecto ambiental escolar ( $\mathrm{PRAE}^{9}$ ) como una estrategia educativa para la educación ambiental, que de cierta manera, establece pautas de comportamiento sobre aspectos ambientales, como el manejo de residuos sólidos. Para el caso de las instituciones de educación superior (Universidad de la Amazonia, UNAD), sí se estableció la existencia de una política ambiental y un trabajo importante respecto al planteamiento del SGA, que si bien tienen como referente teórico la norma ISO 14001, no está estructurado para proceso de certificación.

Respecto a la existencia de sistemas agropecuarios con certificación RAS en el departamento del Caquetá, se encontró en la totalidad de los encuestados, la inexistencia de este tipo de estándar como soporte para certificar los sistemas de producción en las fincas. En contraste con las entrevistas realizadas, se encontró que la norma es desconocida en el departamento.

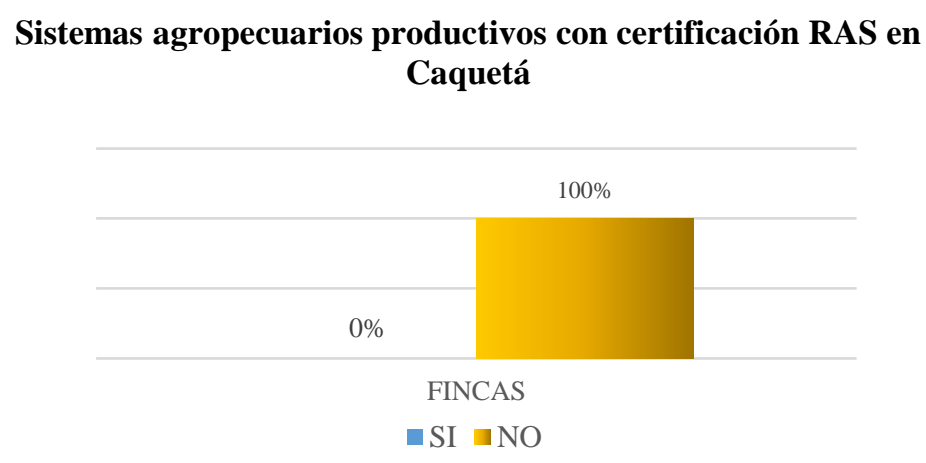

Figura 4. Gráfica de existencia de sistemas agropecuarios productivos con certificación RAS en el departamento del Caquetá.

Cabe resaltar que la mayoría de las fincas productoras encuestadas para este estudio correspondieron principalmente a fincas ganaderas con producción de leche y queso o con cultivos de plátano, yuca y panela en los municipios de San Vicente del Caguán, Florencia, El Doncello y Belén de los Andaquíes.

Ahora bien, la indagación frente a la certificación OHSA 18001 como estándar en la reducción y eliminación de los riesgos en términos de seguridad y salud ocupacional, permitió determinar que la totalidad de las entidades bancarias, las entidades de salud, las entidades de educación y las fincas no existe la aplicación a la existencia de norma. Se estableció que el estándar OHSA, al igual que el RAS, no es conocido por los personajes entrevistados de estas categorías de organizaciones.

Sin embargo, en algunas organizaciones y empresas del sector comercial sí se encontraron certificaciones OHSA, respecto al tema de seguridad y riesgos. Se obtuvieron datos de certificaciones en el ICBF y en las empresas de transporte Las Ceibas y Taxis Verdes. También se identificó en el departamento, la existencia de la empresa Petroservices ambiental dedicada al estudio de impactos ambientales, al asesoramiento empresarial en el tema de riegos y contingencias y a la asesoría frente a estándares internacionales ISO 9001, ISO 14001, RAS y OHSA 18001.

\footnotetext{
${ }^{9}$ Son proyectos que incorporan la problemática ambiental local al quehacer de las instituciones educativas, teniendo en cuenta su dinámica natural y socio-cultural de contexto.
} 


\section{AMAZUNAN}

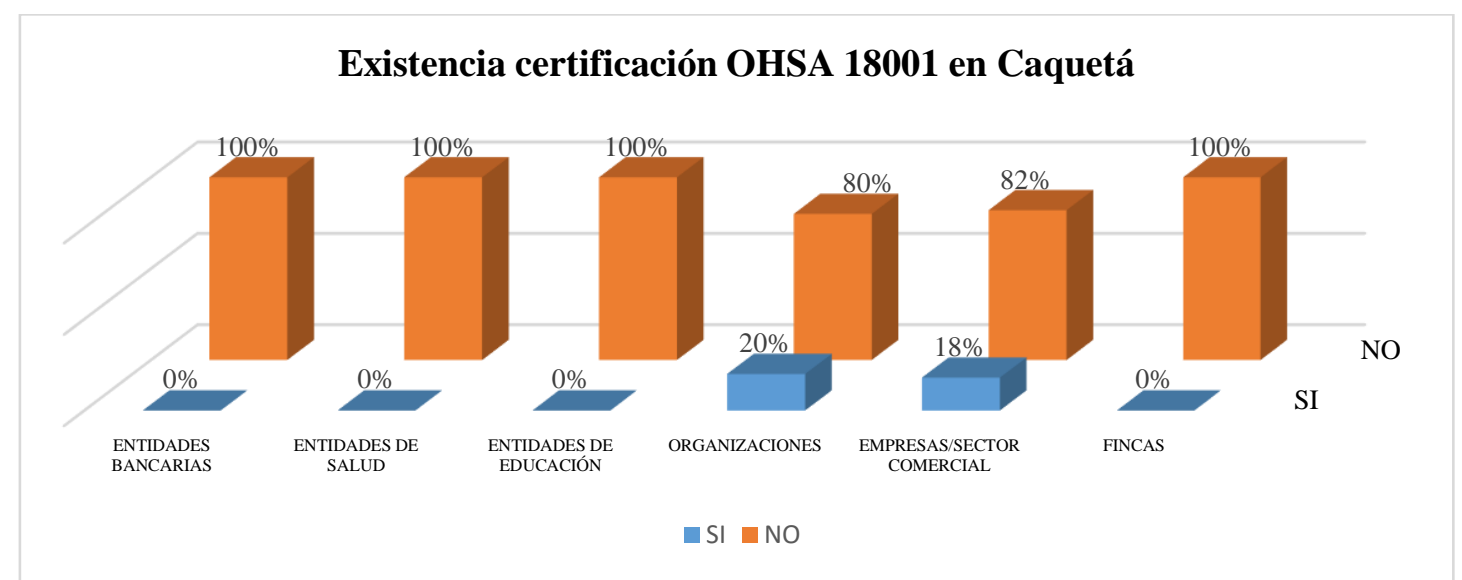

Figura 5. Existencia de certificación OHSA 18001 en el departamento del Caquetá.

\section{Discusión}

El impacto potencial de la estandarización en SGA en las empresas y fincas caqueteñas y economía del país.

Para la dirección de la empresa privada en el departamento del Caquetá, el tema de responsabilidad social en relación con el manejo ambiental y el control de riesgos y seguridad es muy importante, puesto que determina el aporte de la organización en los procesos de conservación ambiental y en la salud de los trabajadores, en cambio, para la administración de las fincas, la visión ambiental no genera una trascendencia tan marcada debido a que los procesos productivos se desarrollan en el ambiente, de manera que los residuos son absorbidos de manera natural y no generan para los propietarios, un impacto ambiental considerable.

Sin embargo, la tendencia de las apreciaciones de ambos grupos de empresarios sobre los procesos de certificación, se orientan a considerar los SGA como un elemento que da valor agregado a los productos y servicios ofrecidos. Los entrevistados en diferentes empresas comerciales y fincas coincidieron que más que el manejo ambiental, la estandarización genera calidad y credibilidad sobre los procesos y productos, es decir, la aplicabilidad de la normatividad concuerda con un mecanismo de valor agregado y generación de ventaja competitiva.

En ese mismo sentido, González (2011) sostiene que actualmente las empresas están sometidas a un ambiente globalizado y a entornos muy competitivos, razón por la cual las pequeñas y medianas empresas (PyMES) en Colombia han comenzado a preocuparse por la realización de una muy buena gestión empresarial.

En Colombia, las PyMES son el grupo predominante de empresas ocupa un 95\% del sector comercial, por eso, el reto para las empresas es implementar sistemas integrados de gestión, en calidad, ambiental y salud ocupacional, bajo los referentes de las normas ISO 9001, ISO 14001 e ISO 18001, dando una orientación de cómo pueden hacerlo y los beneficios que pueden obtener en cuanto a productividad y competitividad se refiere. Para el país, el impacto potencial se resume en el aumento del PIB nacional, pues, según el departamento de investigaciones del Banco Interamericano de Desarrollo, las pequeñas empresas generan el $65 \%$ del empleo industrial en Colombia.

Si bien el análisis de los estándares internacionales en este estudio permitió concluir que la norma busca primordialmente el compromiso de la organización para establecer acciones que permitan contribuir a salvaguardar los recursos del planeta, no puede desconocerse que para los 


\section{AMAZONAS}

empresarios, considerando el efecto de la globalización, el factor primordial es la sostenibilidad económica.

De esta manera, el impacto potencial de las normas sobre las empresas, se traduce en la importancia de consolidar la organización con un enfoque sistémico, con la realización de un direccionamiento estratégico claro que apunte hacia una generación de cultura de calidad, donde exista una mejor organización al desarrollar un sistema estructurado, ordenado, con enfoque a los procesos, que le permita reducir sus costos operativos, generar un nuevo y competitivo ambiente de trabajo y desarrollar la satisfacción total de los usuarios al saber lo que esperan los clientes.

Desde la perspectiva de las fincas, el impacto se puede visualizar desde las tendencias que actualmente se manejan a nivel mundial respecto al mercado de productos ecológicos. Según Espinal, Martínez y Espinosa (2005) el mercado de productos ecológicos se encuentra actualmente en crecimiento entre el $20 \%$ y $25 \%$, cifras que ofrecen un alto potencial para el crecimiento del país. Si bien los autores manifiestan que para la generación de estos productos deben constrastarse los costos de producción versus los costos de mano de obra, se podrían ver compensadas con los mayores precios de este tipo de producto. Los precios de los productos ecológicos tienen una prima o mayor valor frente al convencional. Actualmente, Colombia está viviendo un proceso de transformación leve de algunos productores al sistema ecológico. Las hectáreas certificadas se encuentran en aumento y los consumidores empiezan a demandar este tipo de alimentos, razón por la cual, la certificación de procesos productivos en empresas Caqueteñas con normas internacionales, podrían propiciar un alto impacto potencial respecto a la productividad.

\section{El impacto potencial de la estandarización en SGA en educación}

En el ámbito educativo el reto es aún mayor, particularmente en los procesos de formación, en la proyección social y en los procesos investigativos, dado que es imperante la necesidad de contar con políticas ambientales y diseñar e implementar proyectos de gestión ambiental, puesto que los agricultores de bajos recursos son los menos beneficiados con la estandarización de las normas ambientales, razón por la cual, se requiere que los dueños de fincas trabajen en conjunto de manera sistémica, y que las empresas identifiquen las opciones y requisitos de las normas internacionales y es precisamente, este el reto de la educación, puesto que desde la academia y la investigación se puede informar y formar a la comunidad, dinamizando sistémicamente procesos de gestión ambiental.

Estos estándares ambientales, aunque propenden por mejorar los procesos relacionados con el medio ambiente, pueden generar desigualdades que serán evidentes en los ingresos de quienes están certificados ambientalmente y quiénes no, propiciando la intensificación de las diferencias sociales y de la concentración de la riqueza, en este sentido, Altieri y Nicholls (2000) plantea que el reto histórico de la ciencia agrícola moderna es, por lo tanto, reconcentrar sus esfuerzos en los campesinos y en ecosistemas marginales y asumir su responsabilidad por la prosperidad de la agricultura campesina, lo cual en este estudio se considera que puede ser una oportunidad para que las políticas y estándares ambientales funcionen de manera dinámica vinculando instituciones públicas, privadas y campesinos en pro de dar mayor énfasis al mejoramiento integral de los sistemas agrícolas.

De acuerdo con lo mencionado, el sector educativo está obligado a la búsqueda activa de nuevos tipos de investigación y de estrategias para la gestión ambiental en aras de presentar soluciones de inseguridad alimentaria, degradación ambiental y pobreza, teniendo en cuenta las situaciones, necesidades, y expectativas de empresarios y pequeños propietarios de fincas potencializando los conocimientos y recursos nativos. 


\section{AMAZONANAS}

\section{Los sistemas de gestión y el impacto en la cultura}

La cultura puede ser concebida como el conocimiento adquirido que las personas utilizan para interpretar su experiencia y generar comportamientos (Spradley \& McCurdy, 1975 citado en Herrero, 2002).

En ese sentido, si los modelos de gestión pretenden moldear la realidad haciéndola sistemáticamente controlada a fin de que los sujetos lleguen a un punto en que logren aprehender los procesos, se podría inferir que puede haber un cambio cultural. Es decir, cuando aparece un nuevo modelo de gestión, como resultado de un proceso de acumulación de conocimiento, se producen "cambios de paradigma", aspecto que rompe con algunos elementos de modelos anteriores.

De acuerdo a lo mencionado, González (2011) basado en los aportes de Atehortua (2008) sostiene que los modelos de gestión (que para el caso de los estándares internacionales estudiados en este documento, pertenecen a modelos de control normativo), están fundamentados en la teoría de las relaciones humanas o en la teoría de la cultura organizacional, y por esa razón, se presenta autopoiesis de normas de comportamiento de integrantes de la organización.

En este sentido, se puede deducir que para la implementanción de un modelo que haga modificaciones sobre gestión de los procesos, debe necesariamente haber un cambio cultural, el cual incluya principios y valores que deben estar presentes en cada uno de los integrantes de la organización.

Así pues, el impacto de los estándares no solo transforma los procesos mismos de la organización, las estrategias o los productos, sino que además permean cambios en el comportamiento de los sujetos, produciendo patrones de comportamiento orientados hacia procesos organizados y de calidad.

\section{Conclusiones}

- El lenguaje común de las normas establece que la conservación ambiental depende directamente de una estructura organizativa muy sólida, en la cual la auditoría, la verificación constante de las prácticas, la gestión y las políticas de conservación, son el puente que permite lograr la sostenibilidad de los recursos.

- El panorama de aplicación de estándares internacionales sobre SGA en el departamento del Caquetá es escaso debido mayormente al desconocimiento de la norma por parte de las organizaciones locales.

- Prevalece en las empresas y organizaciones la implementación de políticas ambientales, como una forma de mitigar el impacto de los procesos en la generación de residuos y como un aporte desde el ámbito de responsabilidad social.

- El impacto potencial de las normas sobre las empresas, se traduce en la importancia de consolidar la organización con un enfoque sistémico con la realización de un direccionamiento estratégico claro que apunte hacia una generación de cultura de calidad, donde exista una mejor organización al desarrollar un sistema estructurado, ordenado, con enfoque a los procesos, que le permita reducir sus costos operativos, generar un nuevo y competitivo ambiente de trabajo y desarrollar la satisfacción total de los usuarios al saber lo que esperan los clientes.

- El impacto de los estándares internacionales sobre SGA sobre las fincas se puede visualizar desde las tendencias que actualmente se manejan a nivel mundial respecto al mercado de productos ecológicos, puesto que para el productor certificado, estos 
productos ecológicos tienen una prima o mayor valor frente al convencional, aspecto que podría propiciar un alto impacto potencial respecto a la productividad.

- Desde el ámbito educativo, el impacto se puede consolidar en los procesos de formación, en la proyección social y en los procesos investigativos, dado que es imperante la necesidad de contar con políticas ambientales y diseñar e implementar proyectos de gestión ambiental, para que los agricultores y comunidades de bajos recursos (quienes son los menos beneficiados con la estandarización de las normas ambientales), puedan acceder a la estandarización. El reto de la educación en este aspecto, es dinamizar sistémicamente procesos de gestión ambiental desde la academia y la investigación.

- Los modelos de gestión fundamentados en el modelo gerencial de la teoría de las relaciones humanas o la teoría de la cultura organizacional generan cambios en las normas de comportamiento de integrantes de la organización, es decir, cuando aparece un nuevo modelo de gestión, como resultado de un proceso de acumulación de conocimiento, se producen "cambios de paradigma", aspecto que rompe con algunos elementos de modelos anteriores y genera que los sujetos lleguen a un punto en que logren aprehender los procesos y se presente autopoiesis en las normas de conducta, lo que genera cambio cultural produciendo patrones de comportamiento orientados hacia procesos organizados y de calidad.

\section{Referentes Bibliográficos}

Altieri, M. y Nicholls, C. (2000) Agroecologia. Teoría y práctica para una agricultura sustentable. Programa de las Naciones Unidas para el Medio Ambiente. Red de Formacion Ambiental para America Latina y el Caribe. Mexico. Disponible en: http://www.ambiente.gov.ar/infotecaea/descargas/altieri01.pdf

Arcila, D. (2011). Implementación del sistema de gestión ambiental (SGA) bajo la norma NTC-ISO 14001 en el proceso industrial del arroz en la arrocera la esmeralda S.A, Universidad autónoma de occidente. Santiago de Cali. Colombia. Disponible en: http://bdigital.uao.edu.co/bitstream/10614/1638/1/TAA00772.pdf

Atehortua, F. (2008). Modelos de Gestión. En F. Atehortúa, R. Bustamante, \& J. Valencia, Sistema de gestión integral. Una sola gestión, un solo equipo. Universidad de Antioquia. pp. 14 - 63.

AVIANCA (2011). Informe de responsabilidad social y sostenibilidad. Recuperado de http://www.avianca.com/en/Documents/investor-relations/informacion-sostenibilidad.pdf

Bancolombia (s.f.) Política de gestión ambiental. Recuperado de http://www.grupobancolombia.com/webcorporativa/responsabilidad/pdf/PoliticaGestionAm bientalGrupoBancolombia2014.pdf

Benoit, N. (2011) Diseño para desarrollar un proyecto piloto de la implementación de la norma de agricultura sostenible en Indupalma y en las fincas de palma de aceite, con base en la Rainforest Alliance. Universidad autónoma de occidente. Santiago de Cali. Colombia. Disponible en: http://bdigital.uao.edu.co/bitstream/10614/3206/1/TAA01168.pdf

BBVA (s.f.) (2010). Información de responsabilidad corporativa. Recuperado de http://bancaresponsable.com/informacion-de-responsabilidad-corporativa-2010/pdf/medioambiente.pdf

Espinal, C., Martínez, H. y Espinosa, D. (2005). La cadena de cultivos ecológicos en Colombia. Una mirada Global de su estructura y dinámica 1991-2005. Documento de trabajo 68. Ministerio de Agricultura y Desarrollo Rural Observatorio Agrocadenas Colombia. Disponible en: http://bibliotecadigital.agronet.gov.co/bitstream/11348/4015/1/20051121601_caracteriza cion_ecologicos.pdf

FNA. Foro Nacional Ambiental. (2011). Licencias ambientales y su proceso de reglamentación en Colombia. Bogotá, Colombia. http://library.fes.de/A20135A2-2A18-498A-A120F7E372B41EF2/FinalDownload/DownloadIdA5DBE60D2C8F36A70FB6CA39A77BF2DA/A20135A2-2A18-498A-A120F7E372B41EF2/pdf-files/bueros/kolumbien/08360.pdf 


\section{AMAZÉNAS}

González, S. (2011). Sistemas integrados de gestión, un reto para las pequeñas y medianas empresas. Revista Escenarios. 9 (1). pp 69-89.

Guzmán, D. y Medina, J. (2012) Formulación para la implementación de la norma para la agricultura sostenible de la RAS - sello Rainforest Alliance, para la finca cafetera "La Reina" perteneciente a la comercializadora internacional compañía cafetera agrícola de Santander S.A. en el municipio la Virginia (Risaralda). Disponible en: http://www.udistrital.edu.co:8080/documents/138588/3157083/1.+ARTICULO+CIENTIFI CO+-+SELLO+RAINFOREST+ALLIANCE+FINCA+LA+REINA.pdf

Herrero, J. (2002). ¿Qué es cultura? Recuperado de http://pnglanguages.org/training/capacitar/antro/cultura.pdf

Lannelongue, G., Gonzalez, J., Gonzalez, O. (2011). Esfuerzo y eficacia en los sistemas de gestión medioambiental de empresas certificadas ISO 14001. Tesis doctoral. Universidad de Salamanca, España. Disponible en: http://gredos.usal.es/jspui/bitstream/10366/108986/1/DAEE_Lannelongue_Nieto_G_Esfuer zo_y_eficacia.pdf

Miranda, P., Toro, J. y Agudelo, J. (2010). Evaluación del impacto de la cooperación internacional para el desarrollo en la gestión ambiental. Universidad Nacional de Colombia. Instituto de estudios ambientales IDEA, Universidad de San Buenaventura de Cooperación y Desarrollo. Editorial Kimpress Ltda. Bogotá, Colombia.

Méndez, L. (2009). Normas ISO 14000 como instrumento de gestión ambiental empresarial. Trabajo de grado para obtener el titulo de Maestria en gestión de la calidad. Universidad Veracruzana. México. Disponible en: http://www.uv.mx/gestion/files/2013/01/LORENA-MENDEZORTIZ.pdf

Palacios, B., Gutiérrez, A., Sánchez, M. (s.f.). Nvivo 10. (2013). Una herramienta de utilidad en el mundo de la comunicación. Congreso Nacional sobre Metodología del a Investigacion en Comunicación. Recuperado de http://dialnet.unirioja.es/servlet/articulo?codigo=4230552

Perez, R., Bejarano, A. (2007). Sistema de Gestión Ambiental Serie ISO 14.000. Revista EAN. 62. pp. 69-86.

Red de Agricultura sostenible. (2013). Guía de Interpretación General - Norma para Agricultura Sostenible. Costa Rica. Disponible en: http://www.naturacert.org/documents/SAN-G-201S_Guia_Norma_Agricultura_Sostenible.pdf

Secretaría Distrital de Ambiente. (2014) Documento base para la formulación, concertación, implementación, evaluación, control y seguimiento del plan institucional de gestión ambiental - PIGA. Bogotá, Colombia. Disponible en: http://ambientebogota.gov.co/documents/10157/2426046/Lineamientos+PIGA.pdf

Tobasura, I. (2006). La política ambiental en los planes de desarrollo en Colombia 1990-2006. Revista Luna Azul. 22: p. 8-19. Disponible en: http://lunazul.ucaldas.edu.co/downloads/dbdceba9Revista22_2.pdf

Torres, J. (2009). Evaluación de impacto ambiental y plan de manejo ambiental del proyecto parque lineal Chibunga, Cantón Riobamba, provincia de Chimborazo. Escuela Superior plolitécnica de Chimborazo. Disponible en: http://dspace.espoch.edu.ec/bitstream/123456789/519/1/23T0206TORRES\%20JAIME.pdf

Universidad de América. (2013) Proyecto educativo del programa. Bogotá, Colombia. http://www.uamerica.edu.co/documentos/posgrados/PEP\%20GESTION\%20AMBIENTAL _UNIVERSIDAD\%20DE\%20AMERICA.pdf

Varela, J. (2009). Implantación de un sistema de gestión ambiental basado en el reglamento comunitario EMAS en instalaciones acuariologicas. Universidad da Coruña. España. http://ruc.udc.es/bitstream/2183/7091/1/VarelaSenra.JoseManuel_Tese_1.pdf 